\title{
Sequence of the gtfK gene of Streptococcus salivarius ATCC 25975 and evolution of the $g t f$ genes of oral streptococci
}

\author{
Philip M. Giffard, Donna M. Allen, Carolyn P. Milward, Christine L. Simpson and \\ NicholaS A. JACQUES* \\ Institute of Dental Research, United Dental Hospital, 2 Chalmers Street, Surry Hills, NSW 2010, Australia
}

(Received 7 September 1992; revised 7 December 1992; accepted 1 March 1993)

\begin{abstract}
Many strains of oral streptococci secrete glucosyltransferases (GTFs) that polymerize sucrose into glucans that form an integral part of the plaque matrix on the tooth surface. Recently, we reported the cloning of two closely linked GTF-encoding genes ( $g t f J$ and $g t f K$ ) from Streptococcus salivarius ATCC 25975 as well as the sequence of $g t f J$, which encodes a primer-dependent GTF that synthesizes an insoluble product (a GTF-I). In this communication we report the sequence of $g t f K$, which encodes a primer-dependent GTF that synthesizes a soluble product (a GTF-S), as well as the sequence of a small downstream open reading frame of unknown function. The deduced sequence of GtfK was compared with those of seven other streptococcal Gtfs and an unrooted phylogenetic tree constructed. This analysis suggested that Gtfs with similar product specificities do not form phylogenetic clusters and was consistent with currently accepted phylogenetic schemes. The tree was tested by constructing a series of 'sub-trees' from different blocks of the alignment. Evidence was obtained for recombination events involving $g t f B$ and $g t f C$ from $S$. mutans GS-5, gtfJ and $g t f K$ from $S$. salivarius, as well as the gtfI genes from $S$. downei and $S$. sobrinus. The recombination events between $g t f B$ and $g t f C$, and between the two $g t f I$ genes, were confirmed by examining divergences at silent sites.
\end{abstract}

\section{Introduction}

Members of the mutans and sanguis groups of streptococci, as well as Streptococcus salivarius strains, synthesize extracellular glucosyltransferases (GTFs) that are considered to be important virulence factors in the aetiology of dental caries. The GTFs are highly hydrophilic monomeric proteins of approximate $M_{\mathrm{r}} 160000$ that form glucans by the polymerization of the glucose moiety of sucrose and the release of fructose. It is these glucans that promote dental caries by conferring adherent and anti-diffusion properties on dental plaque (Gibbons, 1968). In most cases the GTFs require a primer glucan for activity. These primer-dependent GTFs either extend the length of, or add branching points to, a pre-existing glucan. It is the nature of the linkages in these glucans that determines their solubility, for those polymers that are predominantly $\alpha-(1 \rightarrow 3)$ -

*Author for correspondence. Tel. 612 2820353; fax 6122820368.

Abbreviation: GTF, glucosyltransferase.

The nucleotide sequence data reported in this paper have been submitted to GenBank and have been assigned the accession number Z11872 (gtfK and orfl). linked are insoluble whilst those that are predominantly $\alpha-(1 \rightarrow 6)$-linked are soluble (Walker, 1978; Rölla et al., 1983; Walker \& Jacques, 1987). The GTFs that produce these glucans are appropriately designated GTF-Is and GTF-Ss respectively.

To date, the sequences of seven streptococcal GTFencoding genes have been published (Table 1). It is clear that all these genes are closely related and that their deduced amino acid sequences share a common domain structure in which the $\mathrm{N}$-terminal two-thirds of the

Table 1. Oral streptococcal GTFs of known sequence

\begin{tabular}{|c|c|c|c|}
\hline Enzyme & Strain & Class & Reference \\
\hline GtfB & S. mutans GS-5 & GTF-I* & Shiroza et al. (1987) \\
\hline GtfC & S. mutans GS-5 & GTF-SI* & Ueda et al. (1988) \\
\hline GtfD & S. mutans GS-5 & GTF-S & Honda et al. (1990) \\
\hline GtfI (dn) & $\begin{array}{l}S . \text { downei } \mathrm{MFe} 28 \\
\text { (formerly } S \text {. sobrinus) }\end{array}$ & GTF-I & Ferretti et al. (1987) \\
\hline GtfI (sb) & S. sobrinus 6715 & GTF-I & Abo et al. (1991) \\
\hline GtfS & S. downei $\mathrm{MFe} 28$ & GTF-S & $\begin{array}{l}\text { Gilmore } \text { et al. } \\
(1990)\end{array}$ \\
\hline GtfJ & $\begin{array}{l}\text { S. salivarius } \\
\text { ATCC } 25975\end{array}$ & GTF-I & Giffard et al. (1991) \\
\hline
\end{tabular}

* These enzymes are reported to synthesize mixtures of soluble and insoluble polymers. 
enzyme, designated the 'catalytic domain', is responsible for the cleavage of sucrose (Ferretti et al., 1987). The Cterminal one-third, on the other hand, will bind to glucan and is known as the 'glucan-binding domain' (Ferretti $e t$ al., 1987; Mooser \& Wong, 1988; Kobayashi et al., 1989). An active-site aspartic acid residue has been located in the catalytic domain (Mooser \& Iwaoka, 1989; Mooser et al., 1991), while the glucan-binding domain is composed of a series of glycine-rich repeat sequences that exhibit similarity to the repeats in the glucanbinding protein from Streptococcus mutans (Banas et al., 1990) as well as the ligand-binding domains in Clostridium difficile toxin A and the lysins from Streptococcus pneumoniae (Wren, 1991).

In this communication, we report the sequencing of $g t f K$, which encodes a primer-dependent GTF-S in $S$. salivarius ATCC 25975 (Pitty et al., 1989), and the comparison of the deduced amino acid sequence of GtfK with seven other Gtfs. A plausible multiple alignment of the central portion of the Gtf sequences was constructed and used to deduce a phylogenetic tree.

\section{Methods}

Strains and growth conditions. Plasmids were maintained in Escherichia coli strain NM522 (Gough \& Murray, 1983) and derivatives of bacteriophage $\lambda$ were grown on $E$. coli strain LE392 (Murray et al., 1977). All $S$. salivarius DNA was derived from $\lambda$-A 33 , which has been described previously (Pitty et al., 1989; Giffard et al., 1991). E. coli cells were routinely grown on Luria Bertani (LB) medium (Miller, 1972) supplemented with ampicillin $\left(100 \mu \mathrm{g} \mathrm{ml}^{-1}\right)$, isopropyl $\beta$-Dthiogalactoside (IPTG) $(1 \mathrm{mM})$, or 5 -bromo-4-chloro-3-indolyl $\beta$-Dgalactoside (X-Gal) $\left(100 \mu \mathrm{g} \mathrm{ml}^{-1}\right)$ as appropriate.

DNA sequence determination. All sequence determinations were carried out using the chain-termination method (Sanger et al., 1977). DNA that was to be sequenced was first subcloned into either or both of the flori-containing phagemids pIBI30 or pIBI31 (IBI corporation), using routine procedures (Maniatis et al., 1982). The subcloned fragments are indicated in Fig. 1. In general, sequencing in the first direction was accomplished using a series of nested deletions constructed by the exonuclease III method of Henikoff (1984), and then confirmed in the other direction using custom-synthesized oligonucleotide primers. However, on occasion, sequencing was carried out in both directions using custom-synthesized primers. Templates were either single stranded or double stranded. Single-stranded templates were prepared using the helper phage M13K07 by the method recommended by Pharmacia for their pTZ series of phagemids, while double-stranded templates were prepared by the method of Birnboim \& Doly (1979) and further purified by ultra-centrifugation through $\mathrm{CsCl}$ gradients (Maniatis et al., 1982). All sequencing reactions were carried out using the Pharmacia T7 sequencing kit according to the manufacturer's instructions. Complete sequences were obtained in both directions.

Sequences were assembled using the IBI Pustell sequence analysis software version 2.03. Database searches were carried out using the ' $m f a$ ' program (Pearson \& Lipman, 1988) in the Australian National Genome Information Service (ANGIS) based at the University of Sydney, Australia.

Multiple alignments and phylogenetic tree constructions. Both were carried out using the 'ClustalV' program (Higgins et al., 1992) in the
ANGIS facility at the University of Sydney. The alignment parameters used are described in the appropriate sections. Tree construction was done using the neighbour-joining method (Saitou \& Nei, 1987). In all cases, positions where there was a gap in any of the sequences were ignored, and branches were lengthened to account for multiple substitutions according to the algorithm of Kimura (1983). Confidence limits were calculated by means of the 'bootstrapping' method of Felsenstein (1985) using 1000 samples.

Divergence analyses. The divergences at silent sites (changes that do not alter the identity of the encoded amino acid) and replacement sites (changes that do alter the identity of the encoded amino acid) for pairs of coding sequences were calculated using the program 'Diverge' available within the Genetics Computer Group (GCG) collection of sequence analysis programs accessed through the ANGIS facility. This program makes use of the algorithm and the 'categories' of nucleotide changes described by Perler et al. (1980).

The input for the analyses were in-frame nucleic acid sequence alignments. The nucleic acid alignments were either derived from the amino acid sequence alignments, or were generated by aligning the nucleic acid sequences directly using the 'Best-Fit' program in the GCG package. The presence of gaps in the alignment, and also the necessity to discern local levels of divergence, required that the analyses be carried out using discrete blocks of alignment. The precise conditions used in each experiment are described in the relevant sections.

\section{Results}

\section{The sequence of $\mathrm{gtfK}$ and the downstream region}

We have previously suggested that $g t f K$, which encodes a primer-dependent GTF-S activity, lies 210 bp downstream from $g t f J$, which encodes a primer-dependent GTF-I activity, on the $S$. salivarius chromosome (Giffard et al., 1991; Fig. 1). As we had only reported the $5^{\prime}$ end of the putative $g t f K$ gene, DNA sequence determination was carried out from the limit of this previously published sequence to the right-hand end of the cloned DNA (Fig. 1). It was found that the beginning of the putative $g t f K$ could be extended to give an open reading frame (ORF) of $4797 \mathrm{bp}$ which encoded a polypeptide of $M_{\mathrm{r}} 176466$ that could be readily aligned with other GTF sequences (see below). The $g t f K$ was also preceded by putative ribosome-binding site and promoter elements (Fig. 2).

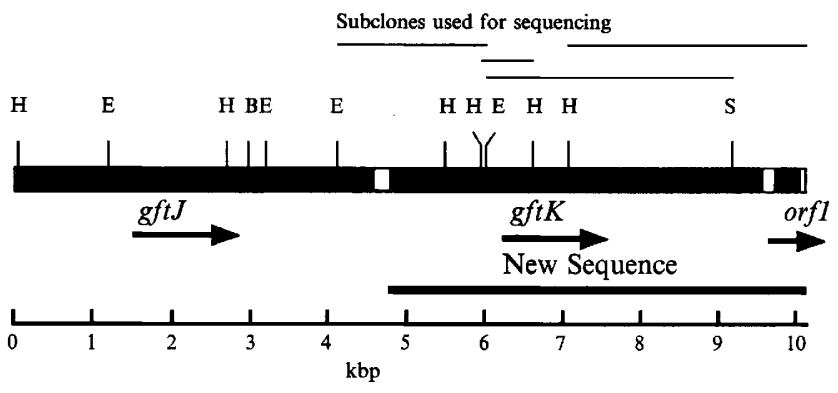

Fig. 1. Physical map of the $g t f J K$ locus, modified from Giffard et al. (1991). Filled areas represent the coding regions and the arrows the direction of transcription. The subclones used for sequencing $g t f K$ and orfl are also shown. H, HindIII; E, EcoRI; B, BgIII; S, SacI. 
The C-terminal third of all sequenced GTFs is composed of a complex series of repeated sequences that have been shown to be associated with glucan-binding. These repeats have been divided into four classes (A-D) (Ferretti et al., 1987; Gilmore et al., 1990; Giffard et al., 1991) although the different classes do exhibit a certain level of similarity. Of these classes, the ' $A$ ' repeat has been found in multiple copies in all GTFs sequenced to date. The GtfK sequence was no exception as it contained seven ' $A$ ' repeats, of which five were immediately followed by sequences that showed similarity to the ' $D$ ' repeats of GtfJ and thus have been classified accordingly. The sequences of the repeats in GtfK and the location of the repeat classes in all known GTF sequences are shown in Fig. 3.

The GTFs are secreted enzymes and so would be expected to possess cleavable $\mathrm{N}$-terminal signal sequences. Ferretti et al. (1987) have carried out Nterminal sequence analysis on the mature secreted form of Gtf-I from $S$. downei and have shown that a signal sequence cleavage site exists downstream of a hydrophobic region that follows von Heijne's ' $-1,-3$ rule' (von Heijne, 1983). The GtfK sequence has an homologous hydrophobic stretch of amino acids followed by two alanine residues that occur at the ' $-1,-3$ ' sites in GtfI and so is also likely to be cleaved at this position (Fig. 4).

\section{An ORF of unknown function follows gtfK}

The sequenced DNA also defined another ORF $141 \mathrm{bp}$ downstream from $g t f K$ that we have designated orf 1 (Fig. 1). This ORF is $345 \mathrm{bp}$ in length and is preceded by putative ribosome-binding site and promoter elements (Fig. 2). It defines a highly hydrophobic polypeptide of $M_{\mathrm{r}} 12884$. The deduced amino-acid sequence was used to search available sequence databases. While no examples of clear similarity were found, the sequence did show low levels of similarity with a number of membraneassociated proteins (data not shown), although by the criteria of von Heijne (1983) there was no clear evidence of any cleavable translocation signal sequence. The function of the product of orfl remains unknown.

\section{Multiple alignment and phylogenetic comparison of available Gtf sequences}

In order to investigate the relationship between different Gtf sequences, it was first necessary to construct a plausible multiple alignment of a portion of the eight $\mathrm{Gtf}$ sequences. This was necessary as two regions within the eight sequences aligned extremely poorly (data not shown). Not unexpectedly, the first of these was the Cterminal glucan-binding domain with its apparent large number of duplications and rearrangements (Fig. 3). The second was a region of approximately 200 amino acids immediately following the signal sequence which was found to be highly variable and difficult to align reliably. Omission of both these domains from the ClustalV analysis yielded a multiple alignment of the eight Gtf sequences that contained a relatively small number of gaps. In order to obtain the 'best' alignment possible, the gap penalties were progressively modified. Significant adjustment of the pairwise gap penalty had no effect on the results, so attention was given to adjusting the multiple alignment fixed gap penalty and floating gap penalty, which control the opening of a gap and lengthening of a gap respectively. Progressively altering these gap penalties had only a slight effect on the multiple alignment until certain values were reached, at which point essential gaps were not opened and the multiple alignment became implausible.

Different alignments were tested by building phylogenetic trees using the neighbour-joining method (Saitou \& Nei, 1987) and comparing the bootstrapping confidence limits, topologies and branch lengths. In all plausible alignments, the confidence limits were $100 \%$ for all branch orders except for the shortest, which varied between $75 \%$ and $90 \%$, the topologies were all identical, and branch lengths the same within $5 \%$. By subjective judgment, the alignment obtained by using a fixed gap penalty of 10 and a floating gap penalty of 30 was chosen for further analysis (Fig. 5).

A phylogenetic tree was constructed from this alignment excluding all positions where gaps occurred (Fig. 6). The topology was tested by bootstrapping (Felsenstein, 1985) using 1000 samples. All the branching orders gave a confidence limit of $100 \%$ except for the separation of GtfJ, GtfK and GtfD from the other sequences which gave a confidence limit of $79 \cdot 3 \%$.

Two significant observations could be drawn from the phylogenetic analysis shown in Fig. 6. First, if one excluded the groupings of GtfB/GtfC, and the two GtfIs (both of which are identical over much of their lengths - see below), there was no clustering of enzymes that make similar glucan products. Neither GtfJ and the two GtfIs, which make insoluble products, nor GtfS, GtfD and GtfK, which make soluble products, form natural groups. Since insoluble glucans are primarily $\alpha-(1 \rightarrow 3)$ linked while soluble glucans are $\alpha$-(1 $\rightarrow 6)$-linked (Walker \& Jacques, 1987; Pitty et al., 1989; Gilmore et al., 1990), it can be inferred that different product linkage specificities may have evolved more than once. Secondly, there was little difference between the maximum divergence between sequences from within the mutans group and the divergence between the sequences from $S$. salivarius and the mutans group. This indicated that in the absence of inter-species gene transfer, the existence of multiple 


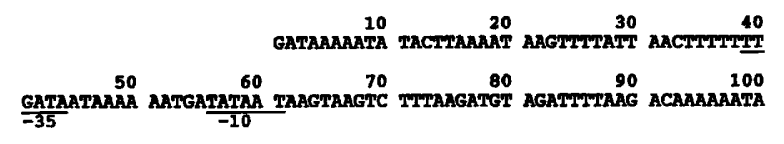

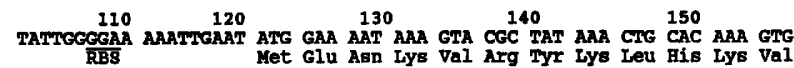

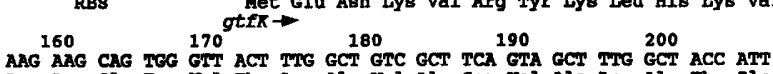
Lys Lys Gla Trp Val Thr Leu Ala val ala ser val Ala Leu Ale thr Ile 210 GET GET AGT GTPT GCC ACA 230 TCT TCA CTC GCT TCE GCA GAA GAA ACA AAT $260 \quad 270 \quad 280 \quad 2902000$ AAC TCT AAT GGA TCA CCT TCA ACA ACT ACE GTC GGG GAA AAT ACA AAT CCT
Asn Ber AEn G1Y Ser Pro Ser Thr Thr Thr VaI Gly Glu AEn Thr ABn Pro $310320330 \quad 340 \quad 350$ GTE GTT GAA AAA GAG GTA GGC ACA ACA ACT GAG GTT GCA AAT ACT TCA AAT Thr Ber Asn $360 \quad 370 \quad 380 \quad 390 \quad 400 \quad 410$ GCC ACA ACA ACA GAG CGA GCA GAR GTT ACT GCT GAC AAA CCA GCT GGA ACG
Ala Thr Thr Thr Glu Arg Ala Glu Val Thr Ala Asp Lys Pro Ala Gly Thr $420 \quad 430 \quad 440 \quad 450 \quad 460$ ACA GTT CAA CCA AAT TCA GGA ACA ACT AGT GAT AGA GCT GCA GCA GTA GAG
Thr Val GIn Pro Asn Ser G1Y Thr Thr Ser ASp Arg Ala Ala Ala Val Glu

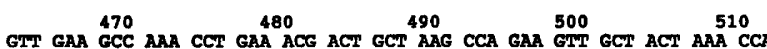
GTI GAA GeC AAA CCT GAA ACG ACT GCT AAG CCA GAA GTT GCT ACT AAA CCA GAA ACA GCT ACA ACA TCA GAA GTC GCT GCA AAT GCT GEA GTA GCT GCT CCA GAA ACA GCT ACA ACA TCA GAA GTC GCT GCA AAT GCT GGA GTA GCT GCT CCA
Glu Thr Ale Thr Thr Ser Glu VaI Ala Ala Asn Nla Gly Val Ala Nla Pro
Ala ACG ACT GAG AAG TCT AAA GAG CTT TCT GAM GCC GAA ATT AAG GCT GCA GTC

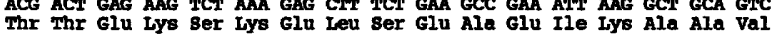
TCA C20 GAT AAT ATT AAA AAA GAA AAA GAT GGT ser Leu Aep Asn Ile Lys lys Glu Iys Asp Gly Lys Tyr Tyr Tyr Leu Leu GAA GAT GGA TCA CAC AAG AAA AAC TTT GCC ATTT A00 GTA AAT GGT CAG GTC $720 \quad 730 \quad 740 \quad 750 \quad 760$

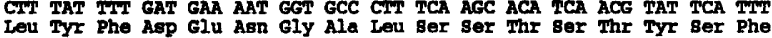

770 ACAN GAM ACA ACA AAT CTA GTC ACT GAT T00 ACA AAR AAT AAT GCT GCC Thr Gln Glu Thr Thr Asn Leu val Thr Asp phe Thr Lys Asn ABn Ala Ale

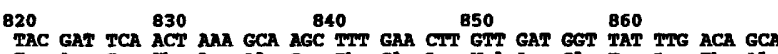
TAC GAT TCA ACT AMA GCA AGC TPT GAA CTT GTY GAT GET TAT TTE ACA GCA
Tyr Asp Ser Thr Lys Ala Ber Phe Glu Leu Val Asp Gly Tyr Leu Thr Ala $870 \quad 880 \quad 990 \quad 900 \quad 910 \quad 920$

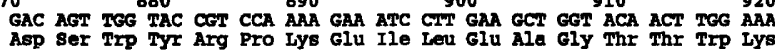
GCE TCA ACT GAA AAA GAT TTP CET CCA C50 T2E ATE TCT TGE TEG CCT GAT GCG TCA ACT GAA AAA GAT TTT CGT CCA CTI TTE ATG TCT TGG TGG CCT GAT
Ala Ser Thr GIU LYS ASP Phe Arg Pro Leu Leu Met Ser TrP TrP Pro Asp AAG GAT 980 CACT GTA GCT TAC TTE AAT TAC ATE ACT 1010 AAG GCT CTT AGC AAC

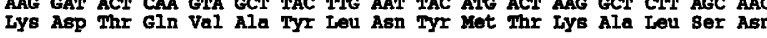
GGT GAA GAA ACA AAA GAT GTC TTT ACG ATT GAM AAT TCT CAA GCT AGC TTE

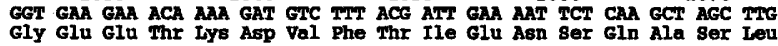
$\begin{array}{lll}1080 & 1090 & 1100\end{array} 1110 \quad 1120$ Asn Ala ala Ala Gin Ile Leu Gln Arg Lys Ile Glu Val Lys Ile Ala Alo 1130
AAT AAG TCA ACA GAC TEG TTE CGT CAG TCA ATC GAG GCT TIT GIT AAA GAC Asn Lys ser thr Aap Trp Leu arg GIn Ser Ile Glu Ala phe val Lys Asp $1180 \quad 1190 \quad 1200 \quad 1210 \quad 1220$

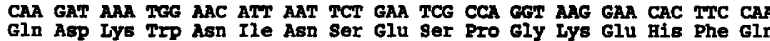
1230
AAA GGT GCG CTT CTT TTT GTG AAC AGC GAT TCA ACT AAA TGG GCT AAC TCT Lys Gly Ala Leu Leu Phe Val Asn Ser Asp Ber Thr LyB TIS Ala Asn Ber 1280
GAT TAT CET AAG CTT AAT CAA ACG GCG ACA AGT TAT ATT AAA AMT CAT AAG Asp Tyr Arg Lys Leu Asn GIn Thr Ale Thr Ser TYY Ile LYS Asn His Lys

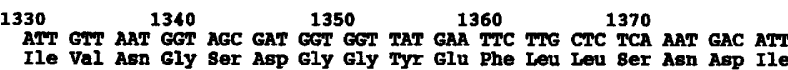
$1380 \quad 1390 \quad 1400 \quad 1410 \quad 1420 \quad 1430$

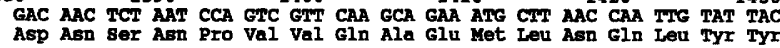
$1440 \quad 1450 \quad 1460 \quad 1470 \quad 1480$ TPT ATG AAC TGG GGA CAA ATC GTC TPT GGT GAT AAG GAC AAG GAT GCT CAC
$1490 \quad 1500 \quad 1510 \quad 1520 \quad 1530$ TPT GAT GGT ATA CGT GTT GAT GCT GTG GAT AAT GTT AGC GTT GAC ATG CTT
Phe Asp Gly Ile Arg Val Asp Ala Val Asp Asn Val Ser Val Asp Met Leu

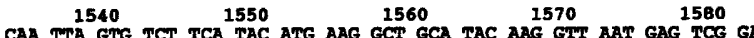
GAA TTA GTG TCT TCA TAC ATG AAG GCT GCA TAC AAG GTT AAT GAG TCG GAA GCT CGT GCC CTT GCE AAT ATC TCT 1610 ATT CTT GAA GCA TGG TCA CAC AAT GAC GCT CGT GCC CTT GCG AAT ATC TCT ATT CTT GAA GCA TGG TCA CAC AAT GAC
Ale Arg AIa Leu Ala Asn Ile Ser IIe Leu GIu Ala TTP Ser His Asn Asp $1640 \quad 1650 \quad 1660 \quad 1670 \quad 1680$ CCA TAT TAT GTG AAT GAG CAC AAC ACA GCA GCT CTT TCT ATG GAT AAT GET
Pro Tyz Tyr Val Asn Glu Hí ABD Thr Ala Ala Leu ser Met Asp ABD GIY

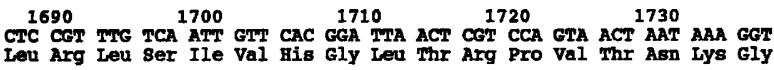
$\begin{array}{lll}1740 & 1750 & 1760 \\ \text { ACA GET GCA CGT AAT GCA TCA ATE AAG GAC CTT ATT AAC GGA GGT TAT TTC }\end{array}$ ACA GET GCA CGT AAT GCA TCA ATG AAG GAC CTT ATT AAC GGA GGT TAT TTC
Thr Gly Ala Arg ABD Ala Ser Met Lys Asp Leu IIe AEn GIY Gly Tyr Phe 1790 CTC $1800 \quad 1810 \quad 1820 \quad 1830$ Gly Leu Ser Asn Arg Ala Glu Val Thr Ser xyr Asp Gln Leu Gly Phe Ale $1840 \quad 1850 \quad 1860 \quad 1870 \quad 1880$ ACC TAC CTC TTT GTS CET GCA CAT EAC TCA GAA GTT CAA ACG GTT ATC GCA $890 \quad 1900 \quad 1910 \quad 19001930$ GAC ATT ATC AGT AAA AAA 1910 GATT GAT CCT ACA ACA GAT GET TTC ACT TTPC 1940 GAC ATT ATC AGT AAA AAA ATT GAT CCT ACA ACA GAT GGT TTC ACT TTC ACA
Asp Ile Ile Ser LYy LYS Ile Asp Pro Thr Thr Asp Gly Phe Thr Phe Thr 1950 TTA GAT CAA TTE AAA CAA GCT TTC GAT ATT TAT AAT GCT GAT ATG TTTG AAA TTA GAT CAA TTG AAA CAA GCT TTC GAT ATT TAT AAT GCT GAT ATG TTG AAA
Leu Asp GIn Leu Lys Gln Ala Phe Asp Ile Tyr Asn Ala Asp Met Leu LyS $2000 \quad 2010 \quad 2020 \quad 2030 \quad 2040$ GTP GAT AAG GAA TAT ACE CAT TCA AAT ATT CCT GCT GCC TAT GCT TrG ATG
Val Asp Lys Glu TyT Thr His Ber Agn Ile Pro Ala Ala TyT Ala Leu Met CIT CAA ACA ATE GET GCE GCA ACA COT GTP TAT TAT GGA GAT TTE TAT ACE Iou Gla Thr Met Gly Ala Ala Thr Arg Val Tyr Tyr Gly Asp Leu Tyr thr 2100
GAT AAT GET CAA TAC ATG GCT AAG AAM TCA CCA TAC TTTI GAT CAA ATC ACA GAT AAT GGT CAA TAC ATG GCT AAG AAA TCA CCA TAC TTPT GAT CAA ATC ACA
Asp ABn Gly GIn Tyr Met Ala Lys Lys Ser Pro Tyr Phe Asp GIn Ile Thr 2150
ACT CTT CTP AAA GCG CET CCT AAG TAT GTA GCT GGT GGT CAA ACA TCT TAT ACT CTr CTP AAA GCG CGr CCT AAG TAT GrA GCr GGT GGT CAA ACA TCT TAT

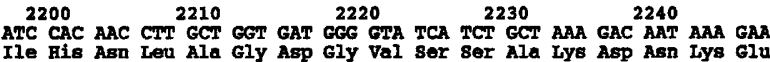
2250 GTT CIT GTC TCT GTC CET TAT GGT CAA GAT 2280 TTE AMT TCT AAG ACA GAT ACA Gal Leu val Ber val Arg Tyr Gly GIn Asp Leu Met Ser LYS Thr ABp Thr

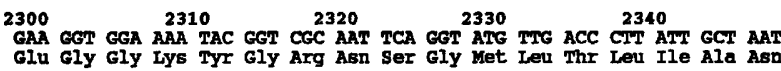

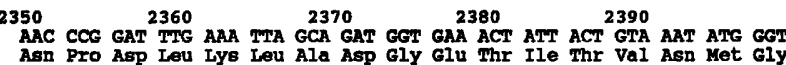
$24002440 \quad 2420 \quad 2430 \quad 2440 \quad 2450$

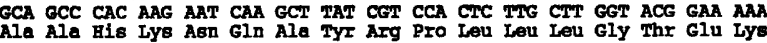
2460
GGT ATT GTG TCA TCA TTE ART GAT TCA GAT ACT AAG ATT GTT AAA TAC ACA Gly Ile val ser ser Leu Asn Asp Ser Asp Thr Lys Ile val Lys Tyr Thr 2510 GAT GCA CAA GGA AAT 2520 GTC TTC ACA GCT GAT GAM 2540 ATC AAA GGC T2T50 GAT GCA CAA GGA AAT CTT GTC TTC ACA GCT GAT GAA ATC AAA GGC TPTT AAG 2560255702500000

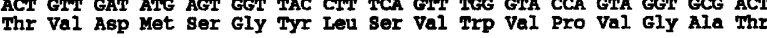
$2610 \quad 2620 \quad 2630 \quad 2640 \quad 2650$ GAC GAT CAA AAC GTT CTT GCT AAA CCA TCA ACA AAA GCC TAT AAA GAA GGG
Asp Asp GIn Asn Val Leu Ala LYS PTO Ber Thr LYB Ala TYY LYS Glu Gly $2660 \quad 2670 \quad 2680 \quad 2690 \quad 2700$ GAT AAG GTP TAT AGC TCT TCT GCA GCA CFT GAA GCA CAA GTG ATT TAC GAA
Asp Lys Val Tyr Ser ser ser Ala Ala Leu GIu Ala Gln Val Ile Tyr Glu $2710272720 \quad 2730 \quad 2750$ GGC TTC TCA AAC TTC CAA GAC TPT GTC AAA GAG GAT AGT CAA TAC ACT AAC
Gly Phe Ber ABn Phe Gln Asp Phe VaI Lys Glu ABp Ser GIn TYY Thr ABD 2760
2770
AAA TTE ATT GCA GCT AAC GCA GAC CTC TTC AAG TCA TGG GET ATT ACG TCA AAA TTG ATP GCA GCT AAC GCA GAC CTC TTC AAG TCA TGG GGT ATT ACG TCA
LyB Leu Ile Ala Ala Asn Ala Asp Leu Phe LyS Ser TIP Gly Ile Thr Ser $2810 \quad 2820 \quad 2830 \quad 2840 \quad 2850$ TrT GAG ATT GCA CCT CAA TAT GTC TCA TCT AAG GAT GEC ACT TTC CTT GAT
Phe Glu Ile Ala Pro Gln Tyr VaI Ser Ber LyS ASP Gly Thr Phe Leu ABP

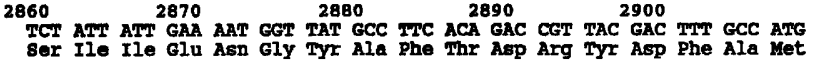


$2910 \quad 2920 \quad 2930 \quad 2940 \quad 2950 \quad 2960$ AGC AAA AAC AAT AAA TAC GGT TCA AAA GAA GAC CTT CGT GAT GCC CTC AAG
Ber LyS ABD ABn LYB TYr GlY Ser LyB Glu Asp Leu Arg Asp Ala Leu LyB $2970 \quad 2980 \quad 2990 \quad 3000 \quad 3010$

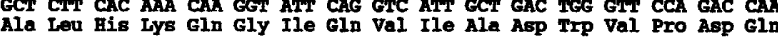
$3020 \quad 3030 \quad 3040 \quad 3050 \quad 3060$ TTG TAC ACC CTT CCA GGT AAA GAA GTA GTG ACT GCT ACG CGT ACT GAT ACA
Leu Tyr Thr Leu Pro Gly Lys Glu Val Val Thr Ala Thr Arg Thr Asp Thr

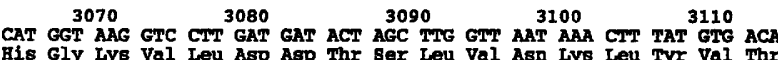
$\begin{array}{lllll}3120 & 3130 & 3140 & 3130 & 3160\end{array}$ AAT ACC AAG TCA TCA GGT AAT GAT TTC CAA GCC CAG TAC GGT GGT GCC TTC
ABn Thr LYS Ber Ser GIY Asn ASp Phe GIn Ala GIn Tyr GIY GIY Ala phe

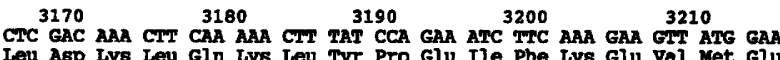$$
3220 \quad 3230 \quad 3240 \quad 3250 \quad 3260
$$

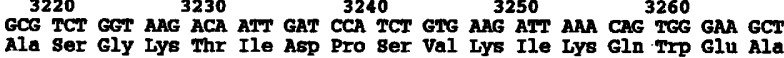
$\begin{array}{lllll}3270 & 3280 & 3290 & 3300 & 3310\end{array}$ AAA TAC TFT AAC GGT ACA AAC ATC CAA AAA CGT GGT TCT GAC TAT GTT CTC
LYB TYY Phe ABn GLY Thr ABn Ile Gln LYB Arg Gly Ser ABP Tyx Val Leu 3320 CAT $3330 \quad 3340 \quad 3350 \quad 3360$

AGC GAT GGT AAA TTG TAC TTC ACC GTT AAC GAT AAA GET ACC TTC CTC CCA $3370 \quad 3380 \quad 3390 \quad 3400 \quad 3410$

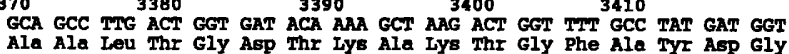

3420 ACA GET GTA ACT TAT TAC 3440 ACA $3450 \quad 3460 \quad 3470$ ACA GGT GTA ACT TAT TAC ACA ACA TCA GGT ACT CAA GCT AAG AGT CAG TTT
Thr Gly Val Thr Tyr Tyr Thr Thr Ser Gly Thr Gln Ala Lys Ber GIn Phe GTA ACT TAT AAC GET AAG CAA TAC TAC $3500 \quad 3510 \quad 3520$ GTA ACT TAT AAC GGT AAG CAA TAC TAC TPT AAC GAT AAG GGT TAC CTT GTA 3530
ACT GGT GAA CAA ACT ATC GAT GCT TCT AAC TAT TTC TTC TTE CCA AAT GGT
Thr GlY Glu GID Thr Ile Asp Gly Ber Asn Tyr Phe Phe Leu Pro Asn GIY 3580
GTC AATE TTP ACT GAT GGT GTC AGA AAA AAT GCC AAA GET CAA TCA 3620
VTTE GTT

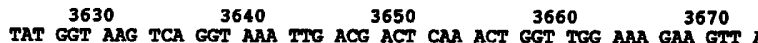
Tyr Gly Lys ser Gly Lys Leu Thr Thr Gln Thr Gly TrP Lys Glu val Thr $3680373690 \quad 3700 \quad 3710 \quad 300$ GTT AAA GAT GAT AGT GET AAG GAA GAG AAA TTC TAC CAA TAC TTC TTC AAG Val Lys Asp Asp sex Gly Lys Glu Glu Lys phe Tyr Gin Tyr phe phe Lys $3730 \quad 3740 \quad 3750 \quad 3760 \quad 3770$ GET GGT ATC ATE GCT ACT GET TTE ACT GAA GTA GAA GGT AAG GAA AAA TAC $3780 \quad 3790 \quad 3800 \quad 3810 \quad 3820$

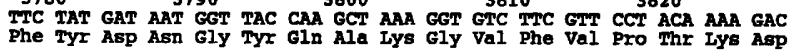
$3830 \quad 3840 \quad 3850 \quad 3860 \quad 3870$ GGC CAT TTA ATG TTC TTC TGT GGT GAT TCA GGC GAA CGT AAG TAC TCA GGC
Gly His Leu Met Phe Phe CYS Gly Asp Ser Gly Glu Arg Lys Tyr Ser Gly

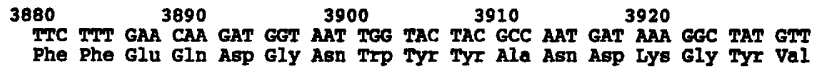

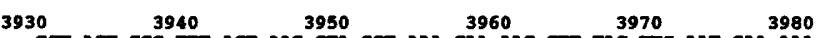

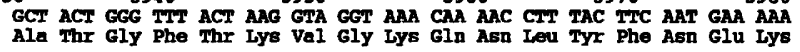

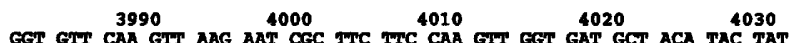
Gly val Gin Val Lys Asn Arg phe phe Gln val Giy Asp Ala thr Tyr Tyx GCA AAT AAC GAA GGT GAC GTT CTS CET GG6 GCG CAA 4070 ACC ATC AAT GGA GAC GCA AAT AAC GAA GGT GAC GTS CTY CET GGT GCG CAA ACC ATC AAT GGA GAC

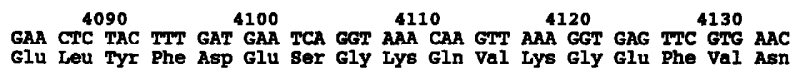
$4140 \quad 4150 \quad 4160 \quad 4170 \quad 4180$ Asn Pro Asp Gly Thr Thr ser Tyr tyr Asp Ala Ile Thr Gly Val Lys Leu $4190 \quad 4200 \quad 4210 \quad 4220 \quad 4230$ GTP GAT ACA TCA CTA GTA GTS GAT GGT CAA ACC TIT AAT GTC GAT GCT AAG val Asp Thr ser Leu val val Aap GIy Gln Thr phe Asn val Asp Ala Lys 4240 GGT GTT GTG ACT AAG GCA CAT ACA CCA GGC TTC T270 ACT ACT GGG GAC AAC Gy val val Thr Lys ala His Thr Pro Gly Phe Tyr Thr Thr Gly Asp Asn AAC TGG TTC TAT GCA GAC TCA 4310 TAT GEA CET AAT GTC ACA GGT GCT CAG GTC AAC TGG TTC TAT GCA GAC TCA TAT GGA CGT AAT GTC ACA GGT GCT CAG GTC
$4340 \quad 4350 \quad 4360 \quad 4370 \quad 4380$

ATC AAT GGT CAA CAC CTC TAC TPT GAT GCT AAT GET CGC CAA GTT AAG GGT

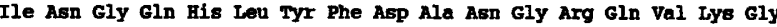
$4390 \quad 4400 \quad 4410 \quad 420 \quad 4430$ GGC TrC GTA ACG AAC ACT GAC GGS AGT CET TCA TFC TAC CAC TGG AAT ACC $4404 \quad 4450 \quad 4460 \quad 4470 \quad 4480$ GGT GAT AAA TTG GPA TCA ACC TTC TTC GCT ACC GET CAT GAT ACA TEG TAT
Gly ABp LYS Leu Val Ser Thr Phe Phe Ala Thr Gly His Asp Arg TrP Tyr $4500 \quad 4510 \quad 4520 \quad 4530 \quad 4540$

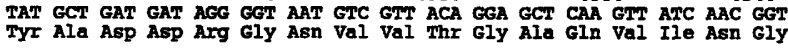
4550
CAA AAA CTC TTC THT GAT ACT GAT GGT AAG CAG GTT 4580 GAA AAA CTC THC THS GAT ACT GAT GGT AAG CAG GTY AAA GGT GCT TPC GCA 4600
ACA AAT GCE AAT GGT AGT CGC TCA TAC TAC CAT TGG AAT ACT GGT ABT AAC

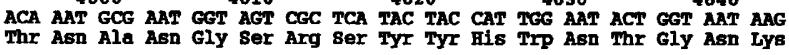
$4650 \quad 4660 \quad 4670 \quad 4680 \quad 4690$ TTA GTA TCA ACC TTC TTC ACT AGT GGA GAT AAC AAC TEG TAT TAT GCA GAT $4700 \quad 4710 \quad 4720 \quad 4730 \quad 4740$ GCC AAG GGT GAA GTT GTT GTT GGC GAA CAG ACG ATC AAC GGT CAA CAC CTT
Ala Lys Gly Glu Val Val Val Gly Glu GID Thr Ile Agn GIY GIn His Leu $4750 \quad 4760 \quad 4770 \quad 4780 \quad 4790$ TAC TPT GAC CAA ACT GET AAG CAA GTG AAG GGG GCA ACT GCT ACG AAT CCT $4800 \quad 4810 \quad 4820 \quad 4830 \quad 40$ GAT GGC TCA ATC TCT TAC TAT GAT GTT CAT ACA GGG GAA AAG GCT ATT AAT $4850 \quad 4860 \quad 4870 \quad 4880 \quad 4890$ CGT TGG GTT AAA ATT CCT TCA GGG CAA TGG GTA TAC TTC AAC GCT CAA GGA
Arg TIP Val Lys Ile Pro Ser Gly GIn TrP Val TyT Phe Asn Ala GIn GIY

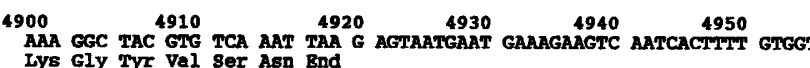

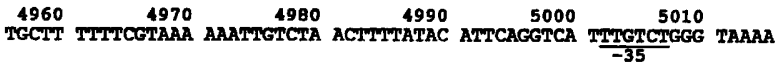

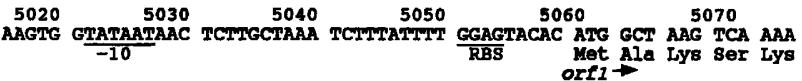
5080
AAT ACT ATT GAA AGT CEG ATA GAT TAC TCE ATY CTC CTE CCA GTT CTC ATA AAT ACT ATT GAA AGT CGG ATA GAT TAC TCG ATT CTC CTE CCA GTT CTC ATA
ABn Thr Ile Glu Ber Arg Ile AEp TyT Ser Ile Leu Leu Pro Val Leu Ile $\begin{array}{lllll}5130 & 5140 & 5150 & 5160 & 5170\end{array}$ TPG CTA CTE GTS GET TTE GTP TCG ATA TPT ATA GCE ACA AAT TTTT GAT TAC 5180
CCA AAG AAT CMT GTG CAA GTC ATG TCT CAG CAA GTA CTA TGG ATT TTT CTI Pro Lys ABn Leu val Gin val Met Ser Gln Gln Val Leu Trp Ile phe Leu $5230 \quad 5240 \quad 5250 \quad 5260 \quad 5270$ GGT TCA GTA CTT GCT TPT GTT GTC ATE TTA TTT AAT ACA GAG TPT CTT TGC Leu TrP $5280 \quad 5290 \quad 5300 \quad 5320$ AAG GTA ACC CCC TGG CTC TAT ATT TPC GGT CTG GTG CTT ATG GTT CTA CCT
Lys Val Thr Pro TrP Leu Tyr Ile Phe Gly Leu val Leu Met Val Leu Pro

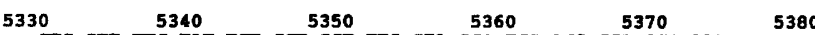

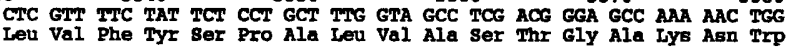
5390
GTA AGC ATT GGA TCT GCG CCC GAA TAA GCC TCAAGCTECA TATPTGATAG TCTE

GCGTAR CCATCATCGA GGATC

Fig. 2. Nucleotide sequences and deduced amino-acid sequences of gtfK and orf1. Potential ribosome binding sites (RBS) and -10 and -35 promoter elements are indicated.

and the mutans group. This indicated that in the absence of inter-species gene transfer, the existence of multiple GTFs might pre-date the divergence of the mutans group from $S$. salivarius.

\section{Evidence for horizontal gene transfer}

Partial recombination events will result in different parts 


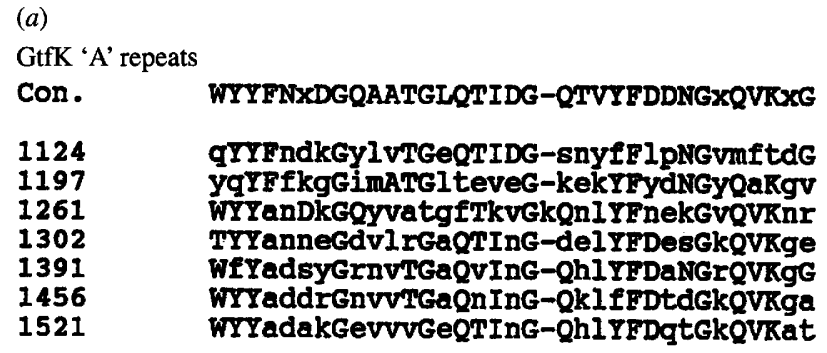

GtfK 'D' repeats

\begin{tabular}{|c|c|}
\hline Con. & VVKNADGTYSKT \\
\hline $\begin{array}{l}1158 \\
1336 \\
1425 \\
1490 \\
1555\end{array}$ & 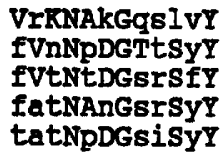 \\
\hline
\end{tabular}

(b)
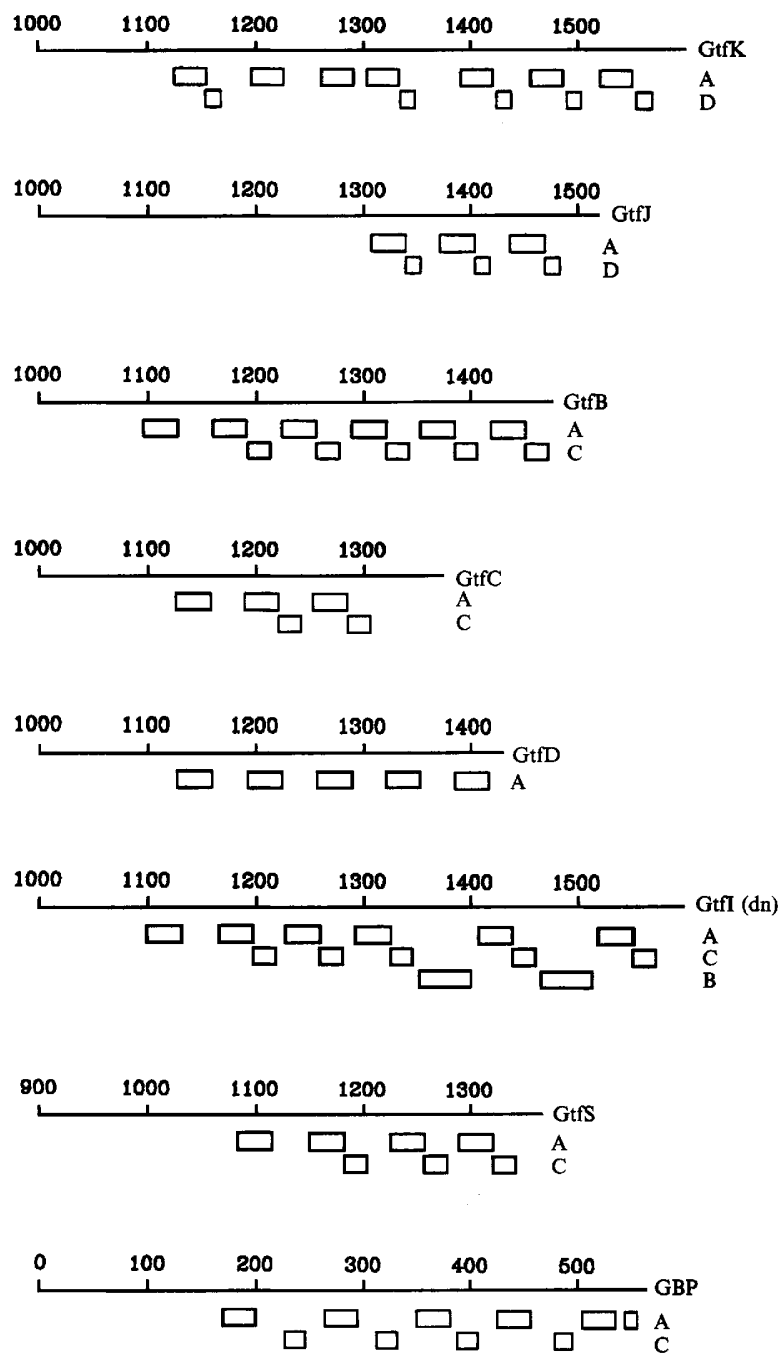

Fig. 3. Repeated sequences from the C-terminal domain of GtfK (a) compared with consensus sequences (Con.) of the 'A' repeat (Gilmore et al., 1990) and ' $\mathrm{D}$ ' repeat (Giffard et al., 1991). The locations of defined classes of repeats in the C-terminal glucan-binding regions are shown in $(b)$. GBP, glucan-binding protein ( $S$. mutans).

\section{$-30 \quad-20 \quad-10$ \\ GtfI (dn) MERNERFKMHRVKKRWVTISVASAA--TM-LASALGASVASA :: : : : :::: ::: ::: : : $:$ : : : : GtfK MENKVRYKLHKVKKQWVTLAVASVALATVIGGSVATSSLASA}

Fig. 4. Comparison of the N-terminal cleavable signal sequence of the GtfI from $S$. downei (Ferretti et al., 1987) with the N-terminus of GtfK. The GtfI has alanine residues at -1 and -3 with respect to the cleavage site while GtfK possesses an homologous site.

of the same gene having different evolutionary histories. In order to test for evidence of such past partial recombination events the region used to constitute the multiple alignment in Fig. 5 was divided into seven blocks of 100 positions and one block of 129 positions (positions where there was a gap in any of the sequences were not counted), and these blocks (Fig. 5) used to construct phylogenetic 'sub-trees' as outlined above. These sub-trees were then examined for localized changes in topology such as the existence of significant groupings in the sub-trees that did not exist in the overall tree (Fig. 6 ), or proportionally large changes in the distance between any two Gtfs.

From the eight sub-trees obtained (Fig. 7) several observations were made. First, the distance between $\mathrm{GtfB}$ and $\mathrm{GtfC}$ decreased dramatically over the length of the sequence analysed. In particular, there was a relatively high divergence between the two sequences in the first two sub-trees, but in the last six sub-trees the sequences were almost identical. This suggested that there had been a recent partial recombination between $g t f B$ and $g t f C$ that involved sequences distal to block 1 . This was plausible since $g t f B$ and $g t f C$ lie next to each other on the $S$. mutans GS-5 chromosome (Hanada \& Kuramitsu, 1988). Secondly, in the phylogenetic tree (Fig. 6), GtfJ and GtfK form a significant group. However, this was only the case in sub-trees 1,3 , and 5 (Fig. 7), although in no sub-trees did the sequences come particularly close. This suggested that there may have been a recombination between $g t f J$ and $g t f K$, but not as recently as between $g t f B$ and $g t f C$. Again this was plausible because $g t f J$ and $g t f K$, like $g t f B$ and $g t f C$, lie next to each other on the chromosome (Giffard et al., 1991). Thirdly, although the GtfI sequences from $S$. downei and $S$. sobrinus showed a high level of similarity throughout the analysed region, there did appear to be proportionally large local changes in the divergence between these two sequences. In particular, the divergence between these two sequences was much greater in sub-trees 1 and 3 than in the other sub-trees (Fig. 7). This finding was somewhat unexpected because $S$. downei (formerly $S$. mutans serotype $h$, then $S$. sobrinus serotype $h$ ) is a monkey isolate (Beighton et al., 1981) while $S$. sobrinus 6715 (formerly $S$. mutans 6715) is a human isolate (Fitzgerald et al., 1968), making recombination 

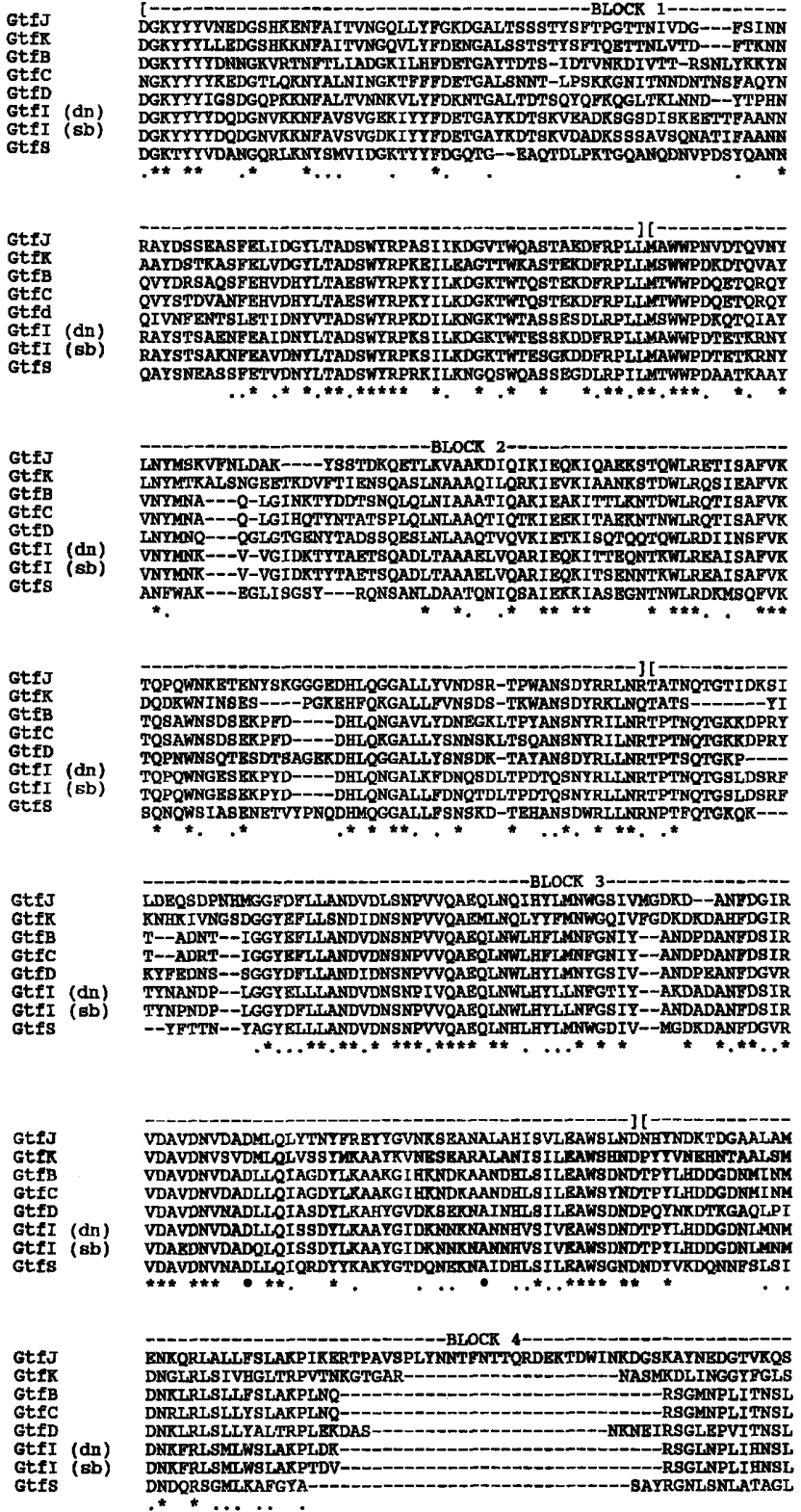

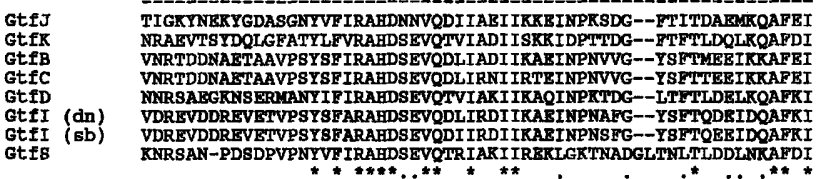
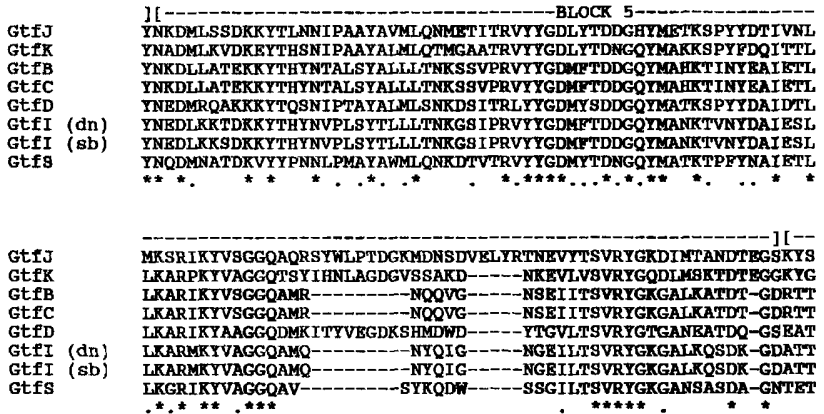
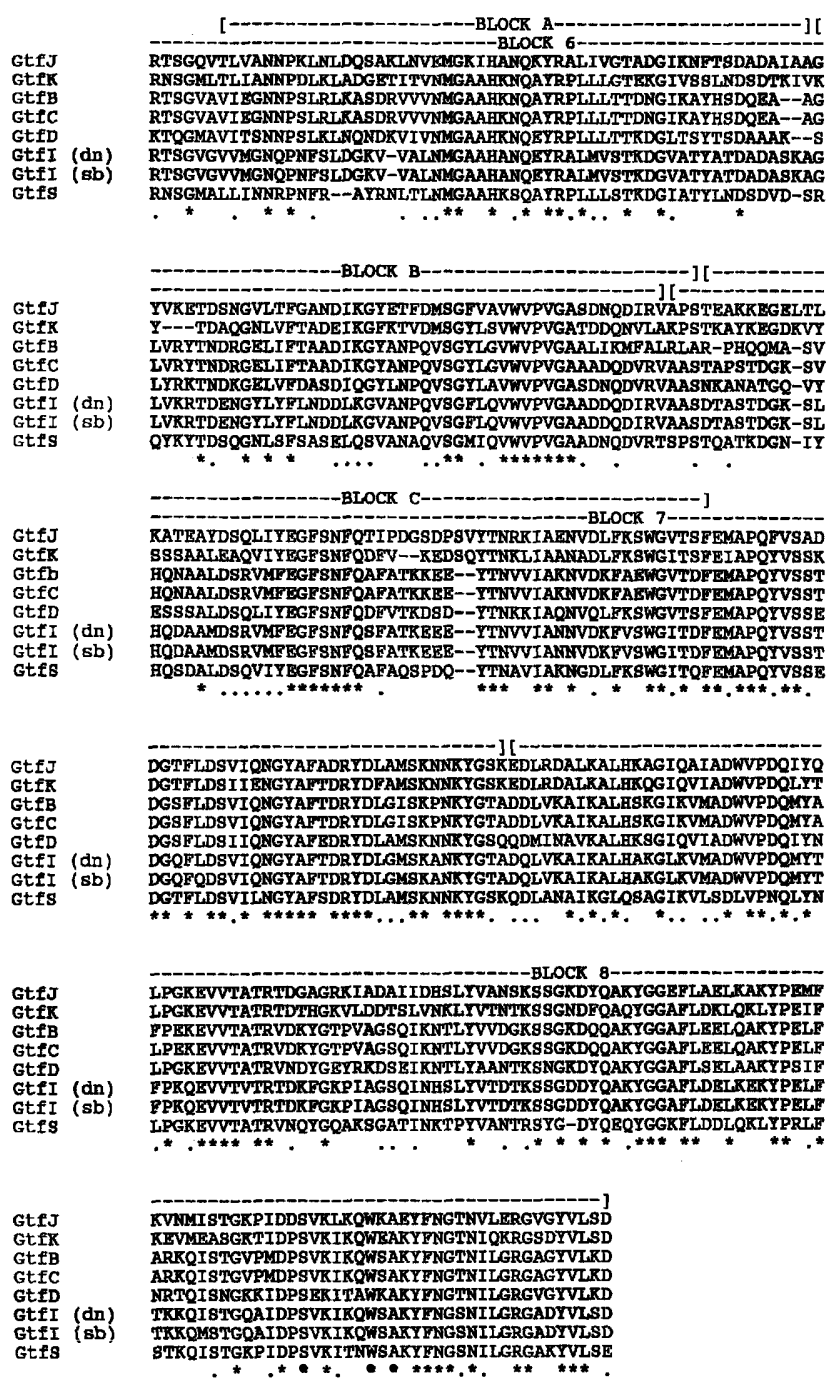

Fig. 5. Multiple alignment of the central portion of the deduced amino acid sequences of the catalytic domains of the eight available Gtfs. The constraints used in constructing the alignment are described in the text. The main blocks (BLOCK 1-BLOCK 8) as well as the three sub-blocks (BLOCK A-BLOCK C) used in subsequent phylogenetic analyses are shown. The residues aligned are: GtfJ, 180-1108; GtfK, 176-1078; GtfB, 166-1037; GtfC, 191-1075; GtfI (dn), 168-1046; GtfI (sb), 162-1035; GtfS, 156-1026; GtfD, 166-1069.

somewhat unlikely. Fourthly, in the overall phylogenetic tree (Fig. 6) GtfB and GtfC formed a significant group with the two GtfIs. However, in sub-tree 6, both GtfB and $\mathrm{GtfC}$ no longer formed a significant group with the two GtfI sequences but instead formed a grouping with GtfD with borderline significance. This suggested that both $\mathrm{GtfB}$ and $\mathrm{GtfC}$ contain sequences that originated from GtfD, implying a small partial recombination between $g t f D$ and the precursor of $g t f B / g t f C$. Again this was plausible because $g t f D$ is found in the same species as $g t f B$ and $g t f C$. Lastly, in no case did GtfS, the GtfIs or GtfJ and GtfK show any sign of forming significant 


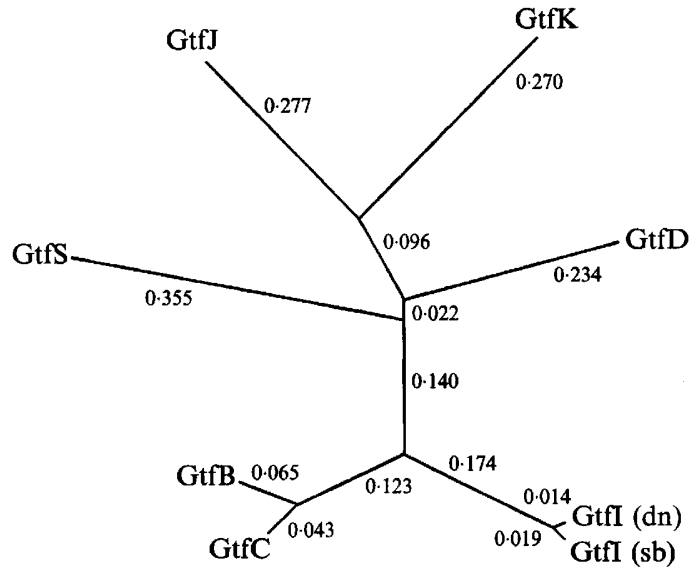

Fig. 6. Unrooted phylogenetic tree constructed from the alignment in Fig. 5 using the neighbour-joining method (Saitou \& Nei, 1987). Relative phylogenetic distances are indicated both numerically and by the length of the connecting lines.

groupings in the sub-trees (Fig. 7) that did not exist in the overall phylogenetic tree (Fig. 6).

\section{Silent-site divergence analysis}

The possibility of partial recombination events between $g t f B$ and $g t f C$ and between $g t f J$ and $g t f K$ as investigated further using the replacement-site and silent-site divergence algorithms of Perler et al. (1980). This strategy depends on the principle that nucleotide identities at codon 'wobble' positions (or silent sites) are not constrained by the functional requirements of the gene product, so if two related but un-recombined genes are compared, they will exhibit a relatively constant level of silent-site divergence over their entire lengths. On the other hand, a recent partial recombination event will result in localized changes in the level of silent-site divergence. These analyses were carried out by determining the nucleotide sequences for blocks of pairwise aligned amino acid sequences within the entire catalytic domain and measuring the divergence of these nucleotide sequences at silent sites and replacement sites. Where possible, the blocks were arbitrarily made 99 nucleotides in length. However, the presence of gaps in the alignments necessitated some flexibility in block size, giving rise to blocks of between 60 and 150 nucleotides in length. In the case of $g t f B$ and $g t f C$, it was clear that the divergence at silent sites was very high at the beginning of the genes, but then decreased significantly around nucleotide 1800 (Fig. 8a), strongly supporting the contention that these two genes had undergone a partial recombination. In the case of $g t f J$ and $g t f K$ (Fig. $8 b$ ), the situation was different. There was no clear evidence of conservation at silent sites, although there may have been slight conservation in the region between residues
500 and 1000 . This region coincides with the first of the sub-trees subjected to phylogenetic analysis, in which GtfJ and GtfK formed a highly significant group. Thus while there was some internally consistent evidence for partial recombination between $g t f J$ and $g t f K$ it was far from clear-cut, indicating that such an event, if it had taken place at all, had not happened recently.

A similar approach was applied to the comparison of the two gtfI sequences, although in this case the very close relationship between the GtfIs meant that the nucleotide sequences could be readily aligned and much larger blocks of sequence used directly as input into the 'Diverge' program. It is clear from this analysis that there were dramatic differences in the silent-site divergence along the length of the two genes (Table 2). This observation supported the notion that there had been a partial recombination between these two sequences, despite their isolation from different bacterial species with different hosts. Also of interest was the large difference between the values of the silent-site and replacement-site divergences despite the high degree of sequence similarity. This would seem to indicate that the majority of the residues within these two enzymes can only undergo a small number of possible changes in order for these changes to remain selectively neutral.

Lastly, this approach was applied to a comparison of GtfD with GtfB and GtfC. In practice, GtfB was excluded from this analysis because of the almost perfect identity of GtfB and GtfC in the analysed region, and also because of a localized reading frame discontinuity in the alignment of GtfB with the other sequences observed

Table 2. Silent-site and replacement-site divergence within the catalytic domains of the GtfIs of S. downei and S. sobrinus

\begin{tabular}{ccc}
\hline \hline & \multicolumn{2}{c}{ Corrected percentage divergence } \\
\cline { 2 - 3 } $\begin{array}{c}\text { Nucleotides from start } \\
\text { of coding region }\end{array}$ & Silent sites & Replacement sites \\
\hline $1-248$ & $69 \cdot 4$ & $8 \cdot 0$ \\
$267-1835$ & $64 \cdot 0$ & $3 \cdot 9$ \\
$1836-2987$ & $3 \cdot 2$ & $0 \cdot 2$ \\
\hline
\end{tabular}

Table 3. Silent-site and replacement-site divergence within blocks $A, B$ and $C$ of the GtfC and GtfD of $S$. mutans

\begin{tabular}{ccc}
\hline \hline \multirow{2}{*}{$\begin{array}{c}\text { Blocks of alignment } \\
\text { (see Fig. 5) }\end{array}$} & \multicolumn{2}{c}{ Corrected percentage divergence } \\
\cline { 2 - 3 } \cline { 2 - 2 } Block A & Silent sites & Replacement sites \\
Block B & $231 \cdot 0$ & 37.9 \\
Block C & $194 \cdot 1$ & $22 \cdot 3$ \\
& 170.2 & 43.0 \\
\hline \hline
\end{tabular}



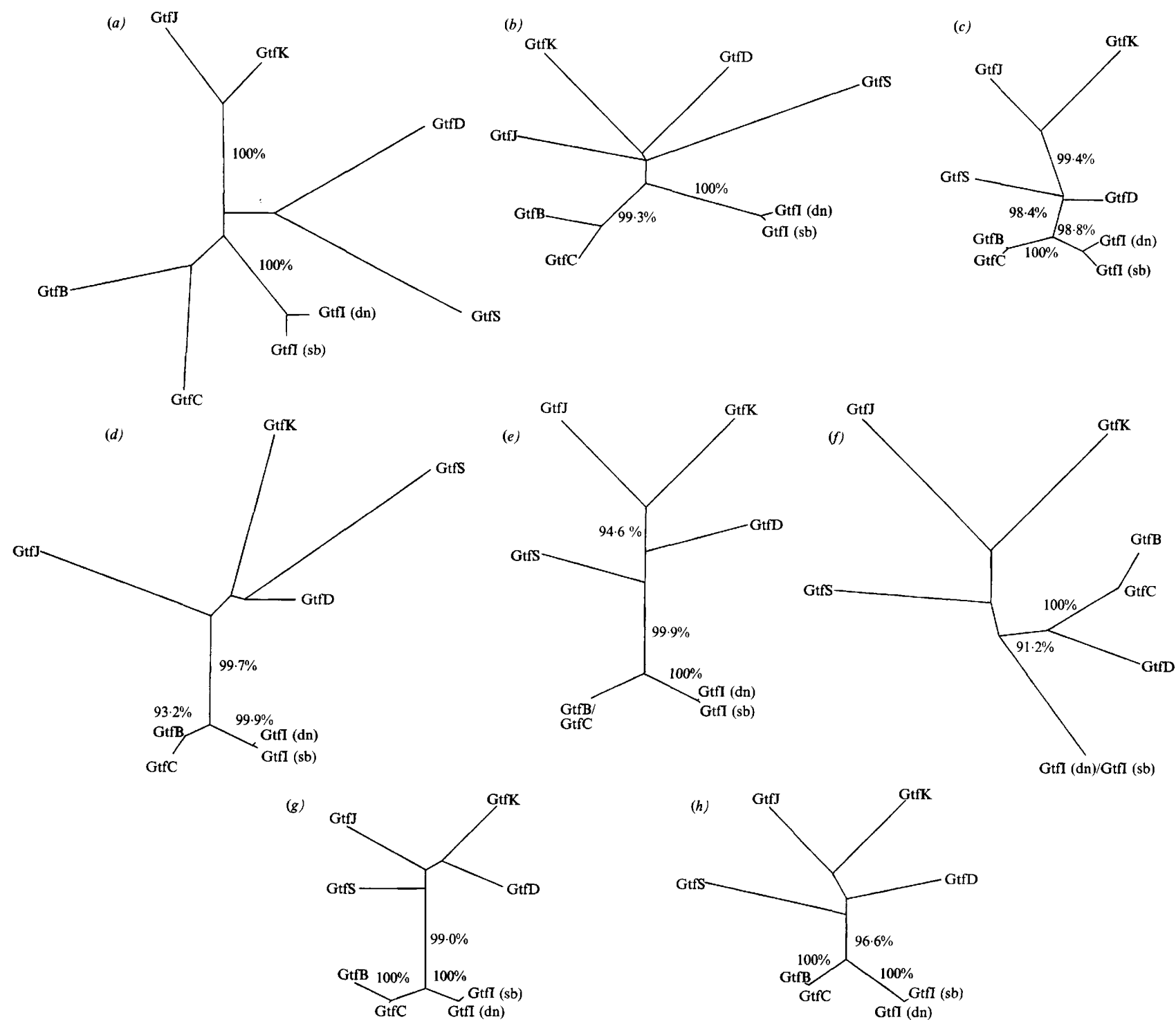

Fig. 7. Phylogenetic 'sub-trees' 1-8 $(a-h)$ derived from blocks 1-8, respectively, of the alignment in Fig. 5. Bootstrapping confidence limits over $90 \%$ are shown.

during the course of this analysis. [This discontinuity encompasses GtfB nucleotides 2397-2450 (numbered from the start codon) and may be explained by either a very recent single nucleotide deletion/insertion event in $g t f B$, or an error in the reported sequence of $g t f B$.] Furthermore, because of the overall relatively high level of divergence of $\mathrm{GtfD}$ from $\mathrm{GtfB} / \mathrm{GtfC}$, the analysis was confined to the region of interest highlighted by sub-tree analysis of block 6 (Fig. $7 f$ ). Three blocks of 150 nucleotides were considered. The central block (block B, Fig. 5) was judged to encompass the amino acid sequences that resulted in the grouping of GtfD with GtfB and GtfC (Fig. 7f), while the two other blocks (block A and block C, Fig. 5) occurred immediately before and after block $B$ and were included for comparison. It was evident from the levels of silent-site divergence in all three blocks that there was no sign of any conservation at silent sites; in fact the analyses indicated a saturating level of substitutions in all three blocks (Table 3). Consequently, if recombination between GtfD and the precursor of GtfB and GtfC had occurred in this region, it was too long ago for conservation at silent sites to be preserved.

\section{Lack of evidence for inter-species transfer of intact gtf genes}

The most common evidence presented for interspecies gene transfer is the presence of closely related genes in unrelated backgrounds, sometimes supported by base composition studies. However, in the case of the gtf genes, all the species are fairly closely related and have 


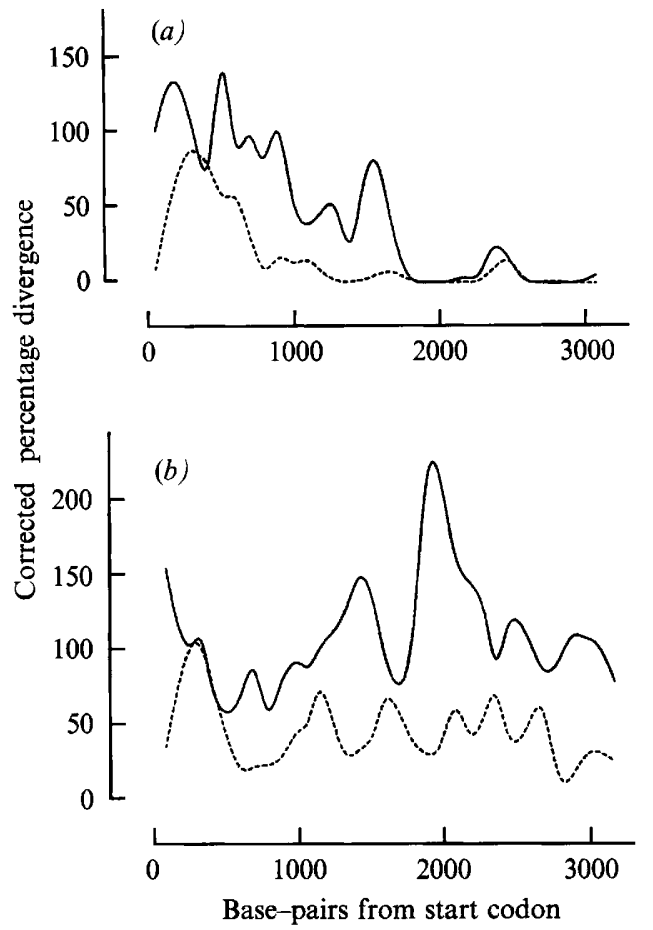

Fig. 8. Divergences of $g t f B$ and $g t f C(a)$ and $g t f J$ and $g t f K(b)$ along the coding sequences for their catalytic domains. Continuous lines represent silent site divergence while dashed lines represent replacement site divergence. Corrected percentage divergences were calculated as described by Perler et al. (1980).

similar base compositions. Therefore, the only approach open to us was to compare the tree in Fig. 6 with the phylogenies derived by DNA hybridization and rRNA sequencing (Schleifer et al., 1984; Ludwig et al., 1985; Schleifer \& Kilpper-Balz, 1987; Bentley, 1991). These latter analyses have consistently found that the mutans group streptococci form a phylogenetically valid (albeit somewhat loose) grouping that does not include $S$. salivarius, that the extent of divergence within the mutans group is not greatly different from the divergence between the mutans group and $S$. salivarius, and that there is a specific close relationship between $S$. downei and $S$. sobrinus. If it were assumed that GtfB, GtfC, and the two GtfIs are all homologues in the strictest sense (i.e. are the products of the same gene duplication/species divergence events), and one does not consider GtfS and $\mathrm{GtfD}$, then the phylogenies derived from DNA hybridization and rRNA sequences are fully consistent with the Gtf phylogenetic tree in Fig. 6. Specifically, the GTF-1 enzymes from $S$. mutans and $S$. downei/S. sobrinus form a fairly loose but valid group that does not include sequences of $S$. salivarius origin, and the GtfIs from $S$. downei and $S$. sobrinus clearly cluster together. Since GtfS and GtfD do not form a group they remain something of a puzzle. It may be that they are not strict homologues and therefore may be the products of different duplication events, having diverged prior to $S$. mutans and $S$. downei becoming separate species. Alternatively, there may be somewhat different selection pressures on GTF-S enzymes, resulting in faster divergence. Whatever the interpretation, there are no groupings in the Gtf phylogenetic tree that do not exist in the rRNA tree, implying that there is no evidence for the transfer of intact $g t f$ genes between the species so far studied.

\section{Discussion}

The sequencing of $g t f K$ completes the sequence analysis of the $g t f J K$ locus as reported by Giffard et al. (1991). The arrangement and spacing of the genes is reminiscent of the $g t f B$ and $g t f C$ of $S$. mutans GS-5, although the gene products have somewhat different functions. Whereas both GtfB and GtfC have been reported to be primerindependent enzymes that synthesize a mixture of soluble and insoluble glucans (Aoki et al., 1986; Hanada \& Kuramitsu, 1988), GtfJ and GtfK are both primerdependent enzymes that synthesize insoluble and soluble glucans respectively (Pitty et al., 1989). Thus, the activity of GtfJ most resembles that of the GtfIs of $S$. downei and $S$. sobrinus while that of GtfK is similar to the GtfD of S. mutans.

GtfK can be clearly aligned with all other Gtf sequences, and contains the putative signal sequence and C-terminal repeats that are typical of this family of enzymes. As with the other Gtfs, the most abundant repeated sequence is the ' $A$ ' repeat while five of these ' $A$ ' repeats are followed by another class of repeat that shows similarity to the ' $D$ ' repeats of GtfJ and therefore has been classified as such. However, the classification of these repeats represents something of a problem in our view, as all of them show a certain level of similarity with one another as well as to the repeats in the ligandbinding domains of the toxins from Clostridium difficile and in the lysins from Streptococcus pneumoniae (Wren, 1991), which are classified according to a completely different scheme. The possibility of a common underlying structure to all these repeat classes is currently under investigation.

Our evolutionary analysis of the eight $g t f$ genes so far sequenced was based on constructing a phylogenetic tree from a highly homologous region in the catalytic domain of these enzymes and then attempting to falsify it by searching for evidence of recombination and/or horizontal gene transfer. The lengths of the sequences aligned meant that a phylogenetic tree with extremely good confidence limits could be constructed. This analysis showed that enzymes with similar activities did not group together and that the existence of multiple Gtfs 
may have pre-dated the divergence of $S$. salivarius from the mutans group.

Partial recombination events were tested for by seeking out localized deviations from the overall relationships between the sequences by constructing a series of subtrees from portions of the alignment and also by carrying out a series of silent-site divergence measurements. From these analyses, clear corroborative evidence was obtained for the partial recombination between $g t f B$ and $g t f C$, and also between the gtfI genes of $S$. downei and $S$. sobrinus. Recombination between $g t f B$ and $g t f C$ was not surprising as these two genes lie next to each other on the $S$. mutans GS-5 chromosome. Furthermore, polymorphisms at this locus have been documented, indicating the potential for rearrangements between these two genes (Chia et al., 1991; Yamashita et al., 1992). In contrast, however, the apparent partial recombination between the two gtf $I$ genes was unexpected. These genes are not only found in different species of streptococci, but the streptococci themselves have different hosts. Gene transfer between the two species would therefore appear at first sight to be unlikely. It may simply be that the two species live in both monkeys and man. Whatever the explanation, these recombination events imply that the distances between GtfB and GtfC and between the two GtfIs in Fig. 6 are an underestimation of the true phylogenetic distances.

The evidence for recombination between the precursor of $g t f B$ and $g t f C$ with $g t f D$ and also for recombination between $g t f J$ and $g t f K$ was much less convincing because it could not be corroborated by silent-site divergence analysis. However, because each group of genes is resident on the chromosome of a specific species, recombination events would not be unexpected. Thus while recombination is probable it remains unconfirmed, and so the divergence between the sequences can be thought of as a minimum not a maximum relative to the time since the sequences diverged.

Apart from the situation with the two gtfI genes, it was difficult to discern any evidence for inter-species gene transfer. However, such events are very difficult to rule out. GTF enzymes are by no means ubiquitous in the genus Streptococcus, yet rRNA sequence studies (Bentley et al., 1991) show no evidence that the mutans group and $S$. salivarius form any sort of natural group. As both species contain $g t f$ genes it is plausible that an ancestral gtf gene(s) was transferred from the mutans group common ancestor to the $S$. salivarius ancestor or vice versa. However, it is not necessary to invoke such an event to rationalize the rRNA phylogenetic tree with the Gtf phylogenetic tree since the Gtf divergence evident both within the mutans group and within $S$. salivarius suggests that such an event must be very ancient if it happened at all. The unravelling of these early events must await a more sophisticated analysis.
This work was supported by a Program Grant awarded by the Australian National Health and Medical Research Council and in part by the Australian Dental Research Fund Inc.

\section{References}

Abo, H., Matsumura, T., Kodama, T., Ohta, H., Fukui, K., Kato, K. \& KaGaWA, H. (1991). Peptide sequences for sucrose splitting and glucan binding within Streptococcus sobrinus glucosyltransferase (water-insoluble glucan synthetase). Journal of Bacteriology $\mathbf{1 7 3}$, 989-996.

aoki, H., Shiroza, T., Hayakawa, M., Sato, S. \& Kuramitsu, H. K. (1986). Cloning of a Streptococcus mutans glucosyltransferase gene coding for insoluble glucan synthesis. Infection and Immunity $\mathbf{5 3}$, 587-594.

Banas, J. A., Russell, R. R. B. \& Ferretti, J. J. (1990). Sequence analysis of the gene for the glucan-binding protein of Streptococcus mutans Ingbritt. Infection and Immunity 58, 667-673.

Beighton, D., Russell, R. R. B. \& Hayday, H. (1981). The isolation and characterization of Streptococcus mutans serotype $h$ from dental plaque of monkeys (Macaca fascicularis). Journal of General Microbiology 124, 271-279.

Bentley, R. W., Leigh, J. A. \& Collins, M. D. (1991). Intrageneric structure of Streptococcus based on comparative analysis of smallsubunit rRNA sequences. International Journal of Systematic Bacteriology 41, 487-494.

BIRnborm, H. C. \& Doly, J. (1979). A rapid alkaline extraction procedure for screening recombinant plasmid DNA. Nucleic Acids Research 7, 1513-1523.

Chia, J.-S., Hsu, T.-Y., Teng, L. J., Chen, J. Y., HahN, L. J. \& YANG, C. S. (1991). Glucosyltransferase gene polymorphism among Streptococcus mutans strains. Infection and Immunity 59, 1656-1660.

FeLSENSTEIN, J. (1985). Confidence limits on phylogenies: an approach using the bootstrap. Evolution 39, 783-791.

FerRetTI, J. J., Gilpin, M. L. \& Russell, R. R. B. (1987). Nucleotide sequence of a glucosyltransferase gene from Streptococcus sobrinus MFe28. Journal of Bacteriology 169, 4271-4278.

Fitzgerald, R. J., Keyes, P. H., Stoudt, T. H. \& Spinell, D. M. (1968). The effects of a dextranase preparation on plaque and caries in hamsters, a preliminary report. Journal of the American Dental Association 76, 301-340.

GiBBONS, R. J. (1968). Formation and significance of bacterial polysaccharides in caries aetiology. Caries Research 2, 164-171.

Giffard, P. M., Simpson, C. L., Milward, C. P. \& Jacques, N. A. (1991). Molecular characterization of a cluster of at least two glucosyltransferase genes in Streptococcus salivarius ATCC 25975. Journal of General Microbiology 137, 2577-2593.

Gilmore, K. S., Russell, R. R. B. \& Ferretti, J. J. (1990). Analysis of the Streptococcus downei gtfS gene, which specifies a glucosyltransferase that synthesizes soluble glucans. Infection and Immunity 58, 2452-2458.

Gough, J. A. \& Murray, N. E. (1983). Sequence diversity among related genes for recognition of specific targets in DNA molecules. Journal of Molecular Biology 166, 1-19.

HANADA, N. \& KURAMitsu, H. K. (1988). Isolation and characterization of the Streptococcus mutans $g t f C$ gene, coding for synthesis of both soluble and insoluble glucans. Infection and Immunity $\mathbf{5 6}$, 1999-2005.

von HeIJNe, G. (1983). Patterns of amino-acids near signal sequence cleavage sites. European Journal of Biochemistry 133, 17-21.

HeNIKOFF, S. (1984). Unidirectional digestion with exonuclease III creates targeted breakpoints for DNA sequencing. Gene 28, 351-359.

Higgins, D. G., Bleasby, A. J. \& Fuchs, R. (1992). CLUSTALV: improved software for multiple sequence alignment. Computer Applications in the Biosciences (CABIOS) 8, 189-191.

Honda, O., Kato, C. \& Kuramitsu, H. K. (1990). Nucleotide sequence of the Streptococcus mutans gtfD gene. Journal of General Microbiology 136, 2099-2105.

KImURA, M. (1983). The Neutral Theory of Molecular Evolution. Cambridge: Cambridge University Press. 
Kobayashi, S., Koga, K., Hayashida, O., NaKano, Y. \& Hasegawa, Y. (1989). Glucan-binding domain of a glucosyltransferase from Streptococcus sobrinus: isolation of a 55-kilodalton peptide from a trypsin digest of glucosyltransferase prebound to insoluble glucan. Infection and Immunity 57, 2210-2213.

Ludwig, W., Seewaldt, E., KilpPer-Bälz, R., Schleifer, K., Magrum, L., Woese, C. R., Fox, G. E. \& Stackebrandt, E. (1985). The phylogenetic position of Streptococcus and Enterococcus. Journal of General Microbiology 131, 543-551.

Maniatis, T., Fitsch, E. F. \& SAMBRoOK, J. (1982). Molecular Cloning: a Laboratory Manual. Cold Spring Harbor, NY: Cold Spring Harbor Laboratory.

MilleR, J. H. (1972). Experiments in Molecular Genetics. Cold Spring Harbor, NY: Cold Spring Harbor Laboratory.

Mooser, G. \& IWAOKA, K. R. (1989). Sucrose 6- $\alpha$-Dglucosyltransferase: characterization of glycosyl-enzyme complex. Biochemistry 28, 443-449.

MoOSER, G. \& WoNG, C. (1988). Isolation of a glucan-binding domain of glucosyltransferase $(1,6-\alpha$-glucan synthase) from Streptococcus sobrinus. Infection and Immunity 56, 880-884.

Mooser, G., Hefta, S. A., Paxton, R. J., Shively, J. E. \& Lee, T. D. (1991). Isolation and sequence of an active-site peptide containing a catalytic aspartic acid from two Streptococcus sobrinus $\alpha$ glucosyltransferases. Journal of Biological Chemistry 266, 8916-8922.

MurRay, N. E., Brammar, W. J. \& MurRay, K. (1977). Lambdoid phages that simplify the recovery of in vitro recombinants. Molecular and General Genetics 150, 53-61.

Pearson, W. R. \& Lipman, D. J. (1988). Improved tools for biological sequence comparison. Proceedings of the National Academy of Sciences of the United States of America 85, 2444-2448.

Perler, F., Efstratiafis, A., Lomedico, P., Gilbert, W., Kolodner, R. \& Dodgson, J. (1980). The evolution of genes: the chicken preproinsulin gene. Cell 20, 555-566.

PitTy, L. J., Giffard, P. M., Gilpin, M. L., Russell, R. R. B. \& JACQUES, N. A. (1989). Cloning and expression of glycosyltransferase activities from Streptococcus salivarius. Journal of Dental Research 68, 1681-1682.
Rölla, G., Ciardi, J. E., Eggen, K. H., Bowen, W. F. \& Afseth, J. (1983). Free glucosyl- and fructosyl-transferase in human saliva and absorption of these enzymes to teeth in vivo. In Proceedings: Glucosyltransferase, Glucans, Sucrose and Dental Caries. Special Supplement to Chemical Sciences, pp. 21-29. Edited by J. R. Doyle \& J. E. Ciardi. Oxford: IRL Press.

SaItou, N. \& NeI, M. (1987). The neighbour-joining method: a new method for reconstructing phylogenetic trees. Molecular Biology and Evolution 4, 406-425.

SANGER, F., Nicklen, S. \& Coulsen, A. R. (1977). DNA sequencing with chain terminating inhibitors. Proceedings of the National Academy of Sciences of the United States of America 74, 5463-5467.

SCHLEIFER, K. H. \& KILPPER-BäLZ, R. (1987). Molecular and chemotaxonomic approaches to the classification of streptococci, enterococci, and lactococci: a review. Systematic and Applied Microbiology 10, 1-19.

SChleIfER, K. H., KilpPer-Bälz, R., Kraus, J. \& Gehring, F. (1984). Relatedness and classification of Streptococcus mutans and 'mutanslike' streptococci. Journal of Dental Research 63, 1047-1050.

Shiroza, T., Ueda, S. \& Kuramitsu, H. K. (1987). Sequence analysis of the gtfB gene from Streptococcus mutans GS-5. Journal of Bacteriology 169, 4263-4270.

Ueda, S., Shiroza, T. \& Kuramitsu, H. K. (1988). Sequence analysis of the gtfC gene from Streptococcus mutans GS-5. Gene 69, 101-109.

WALKER, G. J. (1978). Dextrans. International Reviews in Biochemistry 16, 75-126.

WALKeR, G. J. \& JACQUes, N. A. (1987). Polysaccharides of oral streptococci. In Sugar Transport and Metabolism in Gram-positive Bacteria, pp. 39-68. Edited by J. Reizer \& A. Peterkofsky. Chichester: Ellis Horwood.

WREN, B. W. (1991). A family of clostridial and streptococcal ligandbinding proteins with conserved $\mathrm{C}$-terminal repeat sequences. Molecular Microbiology 5, 797-803.

Yamashita, Y., Bowen, W. H. \& KuRAmitsu, H. K. (1992). Molecular analysis of a Streptococcus mutans strain exhibiting polymorphism in the tandem $g t f B$ and $g t f C$ genes. Infection and Immunity 60, $1618-1624$ 